\title{
Pricing Reactive Power: Some Challenges
}

\author{
Etienne Billette de Villemeur \\ Toulouse School of Economics \\ IDEI \& GREMAQ, \\ University of Toulouse. \\ Email: etienne.devillemeur@univ-tlse1.fr
}

\author{
Chuma Francis Mugombozi \\ École Polytechnique de Montréal, \\ Université de Montréal. \\ Email: francis.mc@ieee.org
}

\begin{abstract}
Distributed generation as attached to market liberalization requires pricing schemes able to deliver the appropriate incentives to market participants. In particular, users should be made responsible for their reactive power. A penalty system fails in that account. One of the major challenges in setting up an appropriate pricing of reactive power consists in identifying its attached costs. Although their peculiar structure might suggest as appropriate a separate treatment of real and reactive power, we argue for a joint approach. In fact, what matters is the total financial burden each user has to support, not the way it is actually computed. A fairness criteria is introduced, according to which no user or group of users should contribute more than its stand-alone cost. When contributions exactly cover total costs, this implies that no user is a burden to the others. We evidence that this criteria is nonsensical if applied to only part of the costs. We conclude by proposing a pricing rule that always complies with our fairness requirements. Its practical implementation is presented and applied to an illustrative example. Tariff penalties for lack of power factor compensation are analyzed for two energy suppliers, one in Europe, the other in North America. They are shown to (possibly) fail to comply with our fairness criteria.
\end{abstract}

Index Terms-Electricity pricing, Reactive power, Game theory, Cost-Sharing.

\section{INTRODUCTION}

With the rise of deregulation and in presence of market players with heterogeneous characteristics, a proper pricing of the different dimensions of electrical services is needed. In particular, in order to send the proper signals to the market, it may appear appropriate to account explicitly for reactive power in pricing energy. Some of the attached challenges are presented in [1]-[8].

Reactive power is almost never explicitly priced. In most cases, tariff penalties are applied to induce utilities or users to display power factors close to one. Yet, absent penalties, system costs attached to reactive power are aggregated to other costs and covered by active power prices. Limitations of penalties have already been discussed and pointed out (See e.g. [9]). In particular, it is plain that penalty rules do not result in any incentives for users to reduce their reactive power consumption, when the latter is below the associated threshold.

While the need for reactive power pricing has been recognized for long, it is still the object of constant research. This

This paper was written while the first author was visiting the department of Economics, at University of Montreal, whose hospitality is gratefully acknowledged. is especially the case in the domain of real-time pricing. In most approaches however, real and reactive power pricing are considered as completely separate problems. Infrastructure, operation and maintenance (O\&M) and other fixed costs are attributed to each dimension in an almost ad hoc way. Production (variable) costs are computed in a separate manner (See again [1]-[4], [8]). It is nevertheless clear that both (i) capacity of the power grid (hence capital and O\&M costs) and (ii) production costs depend upon the amount of exchange and transit of both active and reactive energy. This follows respectively from thermal limitation of the grid and voltage stability requirements.

Following work by Hao and Alexpapapoulos [10], we argue that active and reactive power pricing ought be considered jointly. As evidenced by [10], the costs of providing reactive and real power are so embedded that it is difficult to independently allocate one or the other. This follows in particular from the impossibility to allocate to either service the net capacity of generators to provide MVA power. While the concept of opportunity cost (obtained by considering the case when no real power is consumed, see [9]) is still useful to provide some insights, a proper cost allocation method should consider all the dimensions of the joint-production process. This is all the more true for reactive power pricing. In fact, operational costs attached to reactive power are often very small when compared to others costs. An effort to best allocate this part, by ignoring the others, does not pay. This is the main limit of methods based on nodal equations (see for example [14]). They usually fail to incorporate capital costs, or lack a sound basis for doing so.

By contrast, cost sharing rules are (i) based upon a methodology that accounts for all the costs of the system and (ii) usually derived along a precise axiomatic approach. This may explain why their use is encouraged by [10]. Several contributions have applied cost sharing rules in the context of power system (See, among others, [7]). Unfortunately, it is often done by considering only some dimensions of the power system, hence part of the total costs. Most of the nice properties of the cost sharing rules are lost in this inappropriate usage. In this paper, we are very careful to account for all the costs of the power system. Moreover, we make explicit the properties of the cost-sharing rule we use by coming back to its axiomatic foundations.

Central in our approach is the concept of "fairness", which 
is briefly introduced here (a more detailed analysis is provided in a companion paper [11]). To display clear-cut results, we use a basic radial system with one generation area. It is also assumed that power and voltage are set optimally, and that voltage is within the required range (say $5 \%$ p.u. margin). Even for such a basic system, it appears impossible to decide for the fairness of a reactive power pricing rule without considering the overall cost of the system. Moreover, standard penalty rules may easily yield electricity pricing to fail to comply with our basic fairness requirement. Our companion paper [11] exhibits an allocation method that overcome this weakness. We illustrate its application for a distribution system of industrial loads that display two distinct power factors. We compare it to the outcome of the penalty system of two energy suppliers, one in North America, the other in Europe.

\section{ISOLATING THE COSTS OF REACTIVE POWER}

Absent adequate reactive power pricing, market liberalization may yield important drawbacks, as warned by [12], [13]. Yet, reactive power pricing requires a precise evaluation of the attached costs. An abundant literature covers the issue, which is particularly intricate when generation is distributed (See e.g. [1]-[10], [14]). We illustrate hereafter (some of) the fundamental difficulties attached to isolating the costs of reactive power.

Assume that the system operates under optimal power flow conditions and let circuit elements be delineated in per unit values. The latter implies that transformer rates are removed and the reference voltage is identical over the whole system. Voltage drops as attached to the various positions in the network can easily be tracked. Let $U_{i}$ be the effective node voltage. Active and reactive power losses in transmission lines and transformers depend upon the resistance $R_{i}$ and the reactance $X_{i}$ through the loss coefficients for transmission systems $\gamma_{i}$ and $\gamma_{i}^{*}$ as defined by

$$
\gamma_{i}=\frac{R_{i}}{U_{i}^{2}} \quad \text { and } \quad \gamma_{i}^{*}=\frac{X_{i}}{U_{i}^{2}} .
$$

Observe that, for a properly designed (and well operated) power system, all voltages are within the $5 \%$ p.u. margin. Thus, a characterization of transmission by the coefficients $\gamma_{i}$ and $\gamma_{i}^{*}$ as computed with the reference voltage $U=1$ (rather than with the effective voltage $U_{i}$ ) may provide a good approximation to compute both losses and generated power.

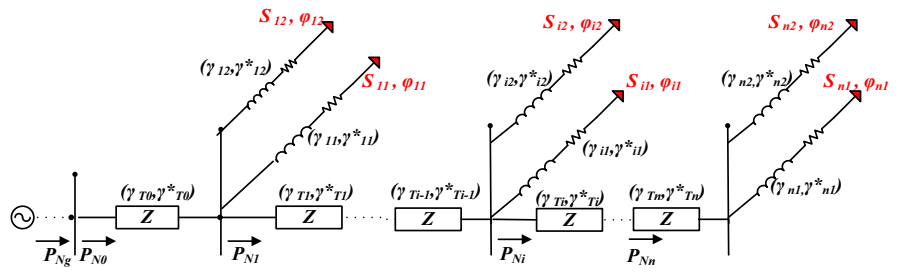

Fig. 1. Electrical system circuit

Consider a radial system with main transmission parameters $\gamma_{T 0}$ and $\gamma_{T 0}^{*}$, distribution system parameters $\gamma_{T i}$ and $\gamma_{T i}^{*}$ and sub-transmission (feeder or service lines system) parameters $\gamma_{i j}$ and $\gamma_{i j}^{*}$, where $i=1 \ldots n$ indexes the buses and $j=1 \ldots m_{i}$ the users per bus. To avoid cumbersome notations, we shall assume that the network is actually a chain and that there are only two users per bus $\left(m_{i}=2\right.$, all $\left.i=1 \ldots n\right)$. The extension of the formulas to the general case is straightforward and left to the reader.

Starting from the downstream bus up to the generation bus, one may compute recursively net real and reactive powers $P_{N i}$ and $Q_{N i}$ to satisfy demand. More precisely, the net real power $P_{N i}$ writes

$$
\begin{aligned}
P_{N i} & =P_{N i+1}+ \\
& +\left[P_{U \text { sers }}+P_{l . T}+P_{\text {l.auxT }}+P_{\text {l.m.T }}+P_{\text {l.l.allT }}\right]_{i},
\end{aligned}
$$

where $P_{N i+1}$ is the net power delivered to the previous (downstream) bus,

$$
\begin{aligned}
P_{U \text { sers }} & =\sum_{j=1}^{2} P_{i j} ; \\
P_{l . T} & =\gamma_{T i} \sum_{j=1}^{2} S_{i j}^{2} ; \\
P_{\text {l.auxT }} & =\sum_{j=1}^{2} \gamma_{i j} S_{i j}^{2} ; \\
P_{\text {l.m.T }} & =2 \gamma_{T i} \sum_{k=1}^{2}\left(\gamma_{i k}^{*} \sum_{j=1}^{2} Q_{j}+\gamma_{i k} \sum_{j=1}^{2} P_{j}\right) S_{k}^{2} ;
\end{aligned}
$$

and

$$
\begin{aligned}
P_{\text {l.l.allT }} & =2 \gamma_{T i}\left(\gamma_{i 1}^{*} \gamma_{i 2}^{*}+\gamma_{i 1} \gamma_{i 2}\right) S_{i 1}^{2} S_{i 2}^{2} \\
& +\gamma_{T i} \sum_{j=1}^{2}\left(\gamma_{i j}^{*}+\gamma_{i j}^{2}\right) S_{i j}^{4} .
\end{aligned}
$$

Equations (2) to (6) define respectively the demand of users, the losses in the main transmission system, the losses in the auxiliary transmission system (feeder), the power needed to cover the losses in the downstream system and the losses attached to this very same losses.

Similarly, the net reactive power $Q_{N i}$ is given by

$$
\begin{aligned}
Q_{N i} & =Q_{N i+1}+ \\
& +\left[Q_{U s e r s}+Q_{l . T}+Q_{\text {l.auxT }}+Q_{\text {l.m.T }}+Q_{\text {l.l.allT }}\right]_{i},
\end{aligned}
$$

where $Q_{\text {Users }}, Q_{l . T}, Q_{\text {l.auxT }}, Q_{\text {l.m.T }}$ and $Q_{\text {l.l.allT }}$ are defined by formulas similar to, respectively, (2) to (6). More precisely, wherever applicable, one should substitute in (2)(6) reactive power $Q$ for active power $P$, active power $P$ for reactive power $Q$; similarly the losses coefficients $\gamma$ and $\gamma^{*}$ are also to be exchanged.

Operation costs can be derived by using the net power at the generation bus, $P_{N g}$. It is fairly reasonable to assume the generation costs to be linear in $P_{N g}$, at least for a given range 

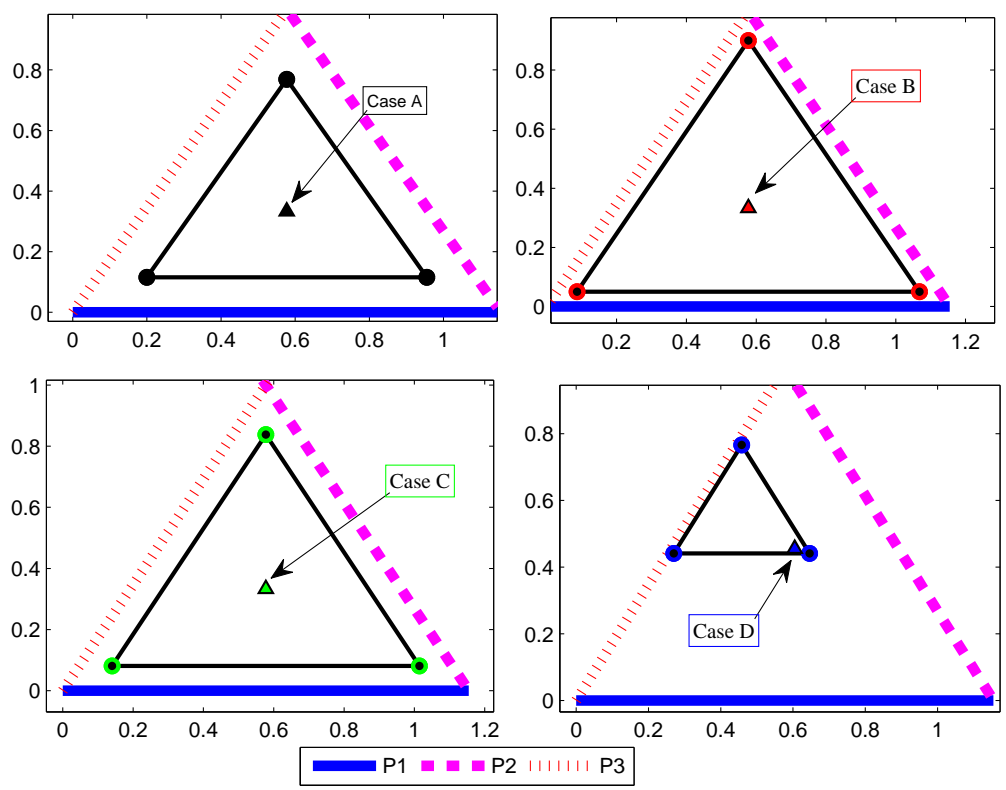

Fig. 2. Illustration of the core area (inner triangle) for cases A, B, C and D listed in Table $I$. Nucleolus allocation is point $\boldsymbol{\Delta}$

of operational parameters. Thus, let the total costs be defined as

$$
\mathcal{C}\left(\mathbf{Z}_{L}\right)=\mathcal{F}+\beta P_{N g},
$$

where $\mathcal{F}$ accounts for all non-variable costs. It is plain that:

- Real and reactive power are deeply embedded. As a result of (5) and (6), the net power at the generation bus $P_{N g}$ is not additively separable in $P$ and $Q$. Thus despite the linearity of $\mathcal{C}\left(\mathbf{Z}_{L}\right)$ in $P_{N g}$, operational costs cannot be decomposed as a sum of costs attached to the real power and costs attached to the reactive power.

- Non-variable costs cannot be unambiguously attributed to active or reactive power either. According to [10], the costs of installed generation capacity may be impossible to split. Moreover, while VAR compensation is directly related to reactive energy support, it does increase real power transfer capability; hence tangibly affects real power transit and the overall costs of the system.

To sum up, a decomposition of the total costs $\mathcal{C}\left(\mathbf{Z}_{L}\right)$ into a sum of costs attached to real power $\mathcal{C}_{P}\left(\mathbf{Z}_{L}\right)$ and costs attached reactive power $\mathcal{C}_{Q}\left(\mathbf{Z}_{L}\right)$ would be nothing but unfounded. We are thus bound to look for a sound pricing approach based upon the sole total costs and yet accounting for both dimensions of the services, namely active and reactive power. We tackle this next. Before to do so, however, a criteria is introduced as to judge for the appropriateness of a pricing rule.

\section{III. "FAIR" PRICING}

\section{A. Fairness Assessment}

Consider a power system with sending end voltage $U$ supplying effective total power $S_{i}$ to users characterized by power factor $\cos \phi_{i}$. Let $Z_{i}\left(R_{i}, L_{i}\right)$ describe the associated users' load. Given the load profile $\mathbf{Z}_{L}=\left(Z_{1}, . ., Z_{n}\right)$, it is possible to define the optimal flow. We assume that the system is run in steady state at optimum and denote $\mathcal{C}\left(\mathbf{Z}_{L}\right)$ the associated total cost. In this paper, we focus on the sole pricing methods such that users exactly cover all the costs. In other words, we consider only cost-sharing methods, as defined by a vector of non-negative contributions $\Psi(\cdot)$ such that

$$
\sum_{i=1}^{n} \Psi_{i}(\mathcal{C}, \mathbf{Z})=\mathcal{C}(\mathbf{Z})
$$

A basic fairness condition requires that no group of users pays more than its stand-alone cost, i.e. what the group would have to pay if its constituents were the sole users of the power system. Formally, let $\mathcal{C}\left(\mathbf{Z}_{G}\right)$ be the "stand-alone" cost of group $G$, where $\mathbf{Z}_{G}$ is the load profile such that $\left\|Z_{j}\right\|=+\infty$, all $j \notin G$ (and $Z_{k}$, all $k \in G$ unchanged). We want that

$$
\sum_{i \in G} \Psi_{i}(\mathcal{C}, \mathbf{Z}) \leq \mathcal{C}\left(\mathbf{Z}_{G}\right), \quad \text { all } G .
$$

Under (9), this is equivalent to

$$
\sum_{i \in G} \Psi_{i}(\mathcal{C}, \mathbf{Z}) \geq \mathcal{C}(\mathbf{Z})-\mathcal{C}\left(\mathbf{Z}_{-G}\right) \quad \text { all } G,
$$

where $\mathbf{Z}_{-G}$ is the load of the complementary group of users. This says that no group $G$ is a burden to the others. Clearly, our fairness requirement is also a necessary and sufficient condition for "voluntary participation". If either (10) or (11) is violated, some users would find advantageous to leave the system and/or to exclude others from accessing it. It follows that our fairness criteria can also be interpreted as a stability requirement.

In the game-theoretic literature, a vector of contributions $\Psi$ is said to be in the core if and only if it satisfies both (9) and (10) 
(or (11)). This concept which goes back to Edgeworth (1881) was formally introduced by Gillies [15]. A necessary (but not sufficient) condition for an allocation to be in the core is that users' contributions $\Psi_{i}$ are higher than (or equal to) marginal costs $\mathcal{C}(\mathbf{Z})-\mathcal{C}\left(\mathbf{Z}_{-i}\right)$. Characterization of the core for radial power systems, including conditions for non-emptiness, may be found in our companion paper [11].

\section{B. Fair Reactive Power Tariffs}

When possible, it is convenient to analyze pricing methodologies by decomposing the cost sharing function $\boldsymbol{\Psi}($.) into two components, namely, one associated to real power and the other to reactive power. Ideally, one would write

$$
\Psi_{i}=\Lambda_{i}+\Phi_{i},
$$

where the sum of $\Lambda_{i} \mathrm{~s}$ is assumed to cover the cost of real power $\mathcal{C}_{P}\left(\mathbf{Z}_{L}\right)$ while the sum of $\Phi_{i} \mathrm{~s}$ is supposed to cover the cost of reactive power service (and capacity requirement) noted $\mathcal{C}_{Q}\left(\mathbf{Z}_{L}\right)$. Formally, this yields two different cost sharing problems to deal with:

$$
\begin{aligned}
\sum_{i=1}^{n} \Lambda_{i} & =\mathcal{C}_{P}\left(\mathbf{Z}_{L}\right), \\
\sum_{i=1}^{n} \Phi_{i} & =\mathcal{C}_{Q}\left(\mathbf{Z}_{L}\right) .
\end{aligned}
$$

On the one hand, it makes very much sense to tackle the two cost-sharing problems in a distinct manner, hence to consider them as completely separate problems. In fact, as already mentioned, the cost structure of reactive power is very peculiar. The variable (or operational) costs attached to reactive power services are usually considered to be very small when compared to the fixed (or capacity) costs. This is to be accounted for when sharing the costs $\mathcal{C}_{Q}\left(\mathbf{Z}_{L}\right)$.

On the other hand, however, there are two fundamental difficulties attached with this approach. First, as already mentioned, it is far from obvious to split even the sole fixed costs $\mathcal{F}$ of the power system into fixed costs attached to real power services $\mathcal{F}_{P}$ and fixed costs attached to the sole reactive power services $\mathcal{F}_{Q}$. Second, the combination of pricing formulas that appear desirable when considering each cost-sharing problem separately, may not be desirable when considering the problem as a whole. Conversely, a sound cost-sharing function $\boldsymbol{\Psi}($. may be such that either or both elements of its decomposition may appear completely awkward.

In particular, one can show that, even if $\boldsymbol{\Lambda}($.$) is deemed to$ be "fair" according to the "core criteria", there is no reason for $\boldsymbol{\Psi}($.$) to inherit this property, unless \boldsymbol{\Phi}($.$) is also "fair"$ according to this same criteria. It is also plain that imposing both $\boldsymbol{\Lambda}($.$) and \boldsymbol{\Phi}$ (.) to obey (10) and (12) or (13) respectively is much more demanding than requiring $\boldsymbol{\Psi}($.$) to obey (10)$ and $(9)$.

In this paper we take the view that what matters is the fairness of pricing "as a whole". We thus look at reactive power pricing through the lens of the (total) cost sharing function $\Psi$.

\section{PROPOSED METHODOLOGY}

\section{A. Core of Cost-Sharing Games: the Power Case}

Cost allocations $\boldsymbol{\Psi}$ are $n$-dimensional vectors. Since we restrict our attention to pricing schemes that exactly cover the costs, we know in addition that $\sum_{i=1}^{n} \Psi_{i}=\mathcal{C}(\mathbf{Z})$. A natural representation of $\boldsymbol{\Psi}$ is thus to be found in the mapping of the cost shares $\left(\Psi_{i} / \mathcal{C}(\mathbf{Z})\right)$ in the simplex $\Delta_{n}$ :

$$
\Delta_{n} \equiv\left\{\mathrm{x} \in[0,1]^{n} \mid \sum_{i=1}^{n} x_{i}=1\right\} .
$$

For illustrations, we set $n=3$ (See circuit in Fig. 3). The simplex $\Delta_{3}$ is of dimension two. More precisely, it is the convex hull of the equilateral triangle of height 1 (See Fig. 2).

An allocation $\Psi$ is a point in $\Delta_{3}$. Each vertex of $\Delta_{3}$ is associated to a user. The distance of the point $\Psi$ to the opposite edge is the cost-share supported by this user. If $\Psi$ is on a vertex, the associated user bears all the costs.

The electrical system is deemed to be very simple as to better display the properties of the problem at hand. As evidenced in our companion paper [11], for the just introduced radial power system, the (set of allocations in the) core is a triangle.

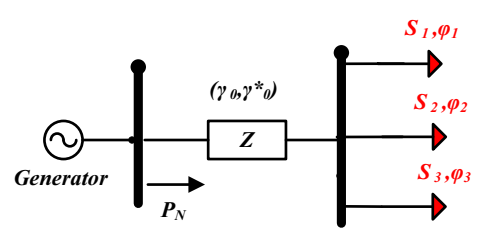

Fig. 3. System of 3 users

Four different user profiles, as characterized by the pair $\left(P_{i}, \cos \phi_{i}\right)$, are considered and listed in TABLE I.

In case $A$, all three users consume the same power $P_{n o m}=$ $270 \mathrm{~kW}$ and display an identical power factor $P F_{h}=0.9$. In case $B$, all three users consume three times more, with an unchanged power factor. In case $C$, users have a consumption identical to the reference case $A$ but their power factor is much lower. More precisely $P F_{l}=0.7$. Case $D$ introduces a double asymmetry across users: they differ in both their real power and their power factor, although total power is identical to the reference case.

The cost function is derived by assuming that the generation costs obey (8) with $\mathcal{F}=52.3 \$ / h$ and $\beta=4.7 \$ / M W h$ (in year basis) and estimating the net power at the generation bus

TABLE I

PARAMETERS FOR ILLUSTRATION OF THE CORE

\begin{tabular}{|c|c|c|c|c|}
\hline cases & $A$ & $B$ & $C$ & $D$ \\
\hline$P_{1}$ & $P_{\text {nom }}$ & $3 P_{\text {nom }}$ & $P_{\text {nom }}$ & $\frac{6}{46} P_{\text {nom }}$ \\
\hline$P_{2}$ & $P_{\text {nom }}$ & $3 P_{\text {nom }}$ & $P_{\text {nom }}$ & $\frac{6}{23} P_{\text {nom }}$ \\
\hline$P_{3}$ & $P_{\text {nom }}$ & $3 P_{\text {nom }}$ & $P_{\text {nom }}$ & $\frac{60}{23} P_{\text {nom }}$ \\
\hline $\cos \phi_{1}$ & $P F_{h}$ & $P F_{h}$ & $P F_{l}$ & $P F_{h}$ \\
\hline $\cos \phi_{2}$ & $P F_{h}$ & $P F_{h}$ & $P F_{l}$ & $P F_{h}$ \\
\hline $\cos \phi_{3}$ & $P F_{h}$ & $P F_{h}$ & $P F_{l}$ & $P F_{l}$ \\
\hline
\end{tabular}




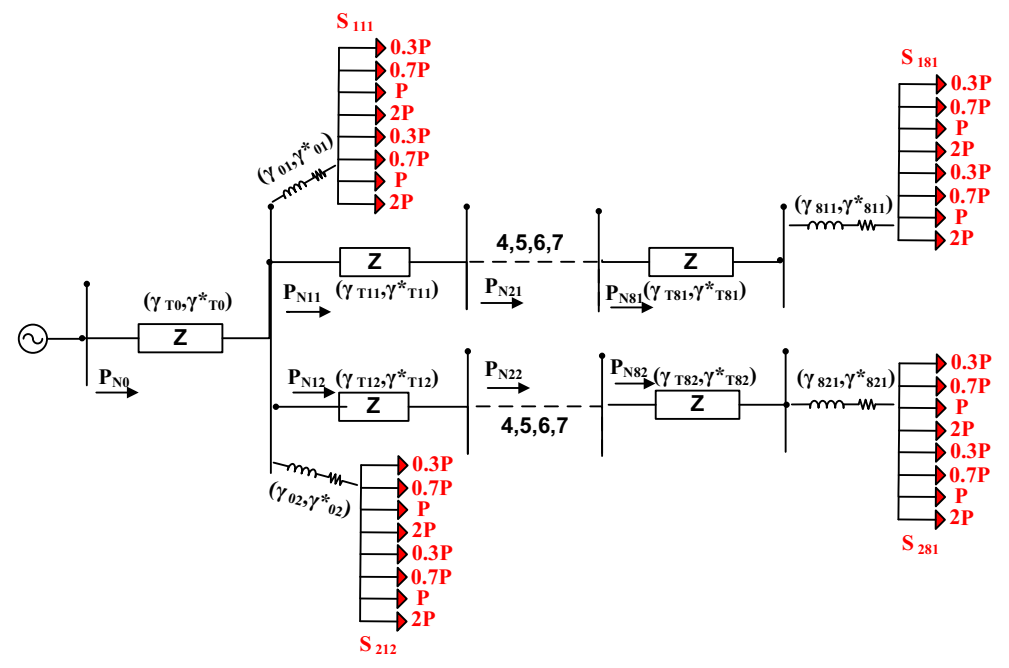

Fig. 4. Distribution system of small industries in p.u

$P_{N_{g}}$. Other parameters include power base $S_{\text {base }}=3 P_{\text {nom }}$ and voltage base $U_{\text {base }}=2.5 k V R M S L L$. The circuit parameter is set to $Z_{T}=(0.28+1.9) \cdot 10^{-3} \mathrm{pu}$. We checked that, in all cases, voltage vary from 1 to $1.03 p u$. Loss coefficients are easily derived.

\section{B. Nucleolus of the Cost-Sharing Game}

If the core is non-empty, a very attractive allocation method is provided by the nucleolus of the cost-sharing game. This concept was introduced by Schmeidler [16] in the general context of a characteristic function game. As Littlechild [17], we consider its application in the particular context of a costsharing game. In loose terms, the nucleolus is the allocation $\Psi$ which maximizes the gains from common use of the power system for the group of users that gains the least. In the particular context of scrutiny, it takes a very simple form detailed in [11]. Indeed, the nucleolus writes:

$$
\begin{aligned}
\Psi_{j}= & {\left[\mathcal{C}(\mathbf{Z})-\mathcal{C}\left(\mathbf{Z}_{-j}\right)\right]+} \\
& +\frac{P_{j}}{\sum_{i=1}^{n} P_{i}}\left[\mathcal{C}(\mathbf{Z})-\sum_{i=1}^{n}\left[\mathcal{C}(\mathbf{Z})-\mathcal{C}\left(\mathbf{Z}_{-i}\right)\right]\right]
\end{aligned}
$$

The latter formula follows from the particular structure of the cost-sharing game which is considered. It does not hold true in general. It has this nice property that, once they paid for their marginal cost, all users $i \in N$ contribute proportionally to their active power to the financing of the remaining deficit. The allocation was computed for cases $A$ to $D$ and plotted in figure 2 . It is the small triangle pointed to by an arrow. We illustrate further the concept in the following section where it is compared to tariffs proposed by different suppliers.

\section{AN ILLUSTRATIVE EXAMPLE}

In this section, we illustrate the possibility to use the concept of "Nucleolus" as a (fair) methodology for pricing electricity. We calibrate distribution system parameters on the city of Sainte-Foy, Québec, Canada, a residential area with about 70000 inhabitants. The distribution system represented in Figure 4 is set to supply electricity to up to approximately 128 small industrial users. Line impedance between buses is $0.0016+0.0012 i$ p.u. for $1.25 \mathrm{~km}$ length $(0.6 \mathrm{kV}$, underground distribution system). Loss coefficients are easily derived. For this illustrative example we set the average power capacity of users to $P=220 \mathrm{~kW}$. More precisely, there are 4 types of users with respectively $30,70,100$ and $200 \%$ of that average power. We set the power factor to 0.7 and 0.9 to highlight possible effects of reactive power variations. Various generation parameters are considered in turn. For each of them, we compute the marginal costs of each users, the price that would follow from the "Nucleolus approach" and the price associated to some actual tariffs as reported by Hydro-Québec, Canada and EDF, France in [18], [19]. More precisely, HydroQuébec displays

$$
\Psi_{i-H Q}=\left\{\begin{array}{l}
\frac{\text { Tariff G: } 50 \mathrm{~kW} \leq P_{i} \leq 100 \mathrm{~kW}}{\Psi_{i}=K_{G}+a_{G} P_{f}+b_{G} P_{i}} \\
P_{f}=\max \left\{P_{\mathrm{i}, \max } ; 0.9 S_{i} ; 0.65 P_{\mathrm{i}, \text { max }- \text { winter }}\right\} \\
\frac{\text { Tariff M: } 100 \mathrm{~kW} \leq P_{i} \leq 5000 \mathrm{~kW}}{\Psi_{i}=a_{M} P_{f}+\tilde{a}_{M} P_{f}+b_{M} P_{i}} \\
P_{f}=\max \left\{P_{i \max } ; 0.9 S_{i} ; P_{\text {nominal }}\right\}
\end{array}\right.
$$

while EDF offers

$$
\Psi_{i-E D F}=\left\{\begin{array}{l}
\frac{\text { Tariff "jaune" }: 42 \mathrm{kVA} \leqslant S_{i} \leqslant 250 \mathrm{kVA}}{\Psi_{i}=K_{J}+\left(a_{J} S_{\max , i}+b_{J} P_{i}\right)} \\
\left.\frac{\text { Tariff "vert" }: 250 \mathrm{~kW} \leqslant P_{i}}{\Psi_{i}=K_{v}+\left(a_{V} P_{\max , i}+b_{V}\right.} P_{i}+c_{V} \mathrm{Q}_{f i}\right) \\
\text { Where: } \mathrm{Q}_{f i}=\mathrm{Q}_{i}-0.4 \mathrm{P}_{i} \text { if }\left(0.4 \leqslant \frac{Q_{i}}{P_{i}}\right) \\
\mathrm{Q}_{f i}=0, \text { otherwise }
\end{array}\right.
$$

We first assume in Fig. 5 that the system is supplied by a 


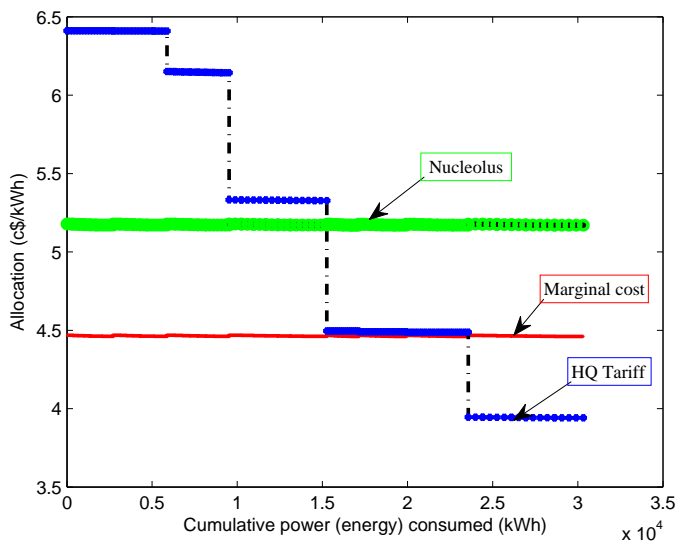

Fig. 5. Illustration with calibration to HQ tariff

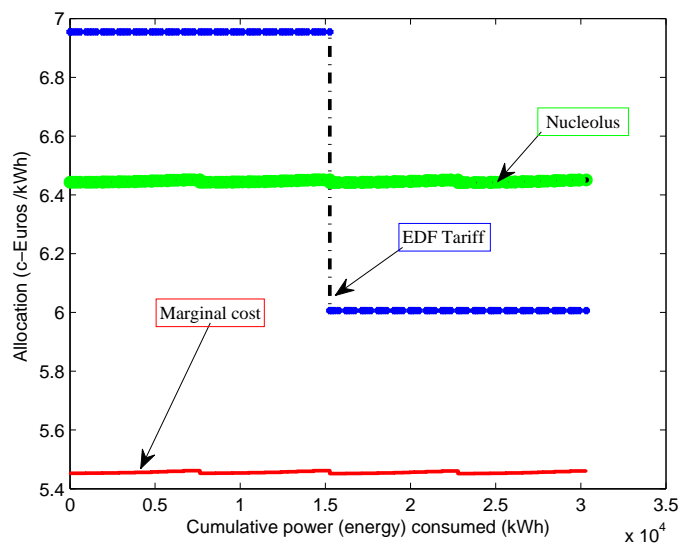

Fig. 6. Illustration with calibration to EDF tariff

natural gas generation plant and we calibrate parameters with the average costs of the energy supplier, Hydro-Québec, in the region of Sainte-Foy. Estimated cost and tariff parameters are set by using [19] and [20]: $\mathcal{F}=215 \$ / h, \beta=45 \$ / M W h$, $K_{G}=12.44 \$, a_{G}=15.54 \$ / k W, a_{M}=13.44 \$ / k W, b_{G}=$ $8.82 c \$ / k W h, b_{M}=4,51 c \$ / k W h$. In Fig. 6 , we apply EDF tariff for the same city. Cost and tariff parameters are set by using [18] and [21]: $\mathcal{F}=300 € / h, \beta=55 € / M W h, a_{V}=$ $120.84 € / k W, b_{V}=5.84 c € / k W h, c_{V}=1.77 c € / k$ Varh.

Clearly, given the Hydro-Québec pricing scheme, and given the assumed conditions, some users appear to pay less than the (marginal) costs they induce on the system (see Fig. 5). In other words, they are a burden to the others. This says that the associated allocation is outside the core. Tariffs are clearly unfair.

\section{CONCLUSION}

In this paper a fairness criteria has been introduced and illustrated. A fair pricing rule, according to the basic criteria introduced, should take into account all costs. Pricing reactive power in isolation appears to be both unrealistic and non sensical. A simulation evidences that actual tariffs as proposed by different suppliers may yield to prices below the marginal cost of some users. An axiomatic approach is proposed that complies with our fairness criteria. We apply it to allocate costs of electricity for industrial users. This analysis may be helpful to assess the pricing methodology in electrical markets.

\section{REFERENCES}

[1] L.Y.C. Amarasinghe, U.D. Annakkage, U.D. Annakkage, (2007), “A Reactive Power Model for a Simultaneous Real and Reactive Power Dispatch", IEEE Power Engeering society meeting, 24-28.

[2] M. L. Baughman, S. N. Siddiqi, (1991), "Real-time pricing of reactive power: theory and case study results", IEEE Transactions on Power Systems, Vol. 6, No.1.

[3] Y. Dai, Y.X. Ni, C.M. Shen, F.S. Wen, Z.X. Han, Felix F. Wu, (1999), "A study of reactive power marginal price in electricity market", Electric Power Systems Research 57 (2001) 41-48.

[4] A.A. El-Keib X. Ma, (1997), "Calculating short-run marginal costs of active and reactive power production", IEEE Transactions on Power Systems, Vol. 12, No. 2.

[5] J. Barquín Gil, T. G. San Rom’n, J. J. Alba Ríos, P. Sanchez Martín, (2000), "Reactive Power Pricing: A Conceptual Framework for Remuneration and Charging Procedures", IEEE Transactions on Power Systems, Vol. 15, No. 2.

[6] F. Li, N.P. Padhy, J. Wang, B. Kuri, (2006), "MW+MVAr-Miles Based Distribution Charging Methodology”, 2006 IEEE Power Engineering Society General Meeting.

[7] X. J. Lin, C.W. Yu, N. Xu, C.Y. Chung, H. Wu, (2006), "Reactive power service cost allocation using Aumann-Shapley method", IEE Proc.Gener. Transm. Distrib., Vol. 153, No. 5.

[8] G. A.Vaidya, N. Gopalakrishnan, (2008), "Cost based Reactive Power Pricing Structure in Restructured Environment", 2008 Power System Technology and IEEE Power India Conference (POWERCON 2008).

[9] M. Muchayi, M.E. El-Hawary, (1999), "A summary of algorithms in reactive power pricing", Electrical Power and Energy Systems, 21, pp. 119-124.

[10] S. Hao, A. Papalexopoulos, (1997), "Reactive Power Pricing and Management”, IEEE Transactions on Power Systems, Vol. 12, No. 1, Feb. 1997.

[11] E. Billette de Villemeur, C.F. Mugombozi, (2008), "Electricity Pricing: Power-Loss Allocation versus Cost-Sharing Methods", mimeo.

[12] J. Kueck, B. Kirby, T. Rizy, F. Li and N.Fall, (2006), "Reactive Power from Distributed Energy", The Electricity Journal, Vol. 19, Issue 10, December 2006, pp. 27-38.

[13] R. J. Thomas, T. D. Mount, R. Schuler, W. Schulze, R. Zimmerman, F. Alvarado, B. C. Lesieutre, P. N. Overholt and J. H. Eto, (2008), "Efficient and Reliable Reactive-Power Supply and Consumption: Insights from an Integrated Program of Engineering and Economic Research", The Electricity Journal, Vol. 21, No. 1, Jan.-Feb. 2008, pp 70-81.

[14] S.N. Khalid, M. W. Mustafa, H. Shareef, and A. Khairuddin, (2008), "A Novel Method for Reactive Power Allocation Using Modified Nodal Equations", Proceedings of the 2007 Australian Universities Power Engineering Conference.

[15] D. B. Gillies, (1959), "Solutions to general non-zero-sum games", in Tucker, A. W.; Luce, R. D., Contributions to the Theory of Games IV. (Annals of Mathematics Studies 40), Princeton: Princeton University Press, pp. 47-85.

[16] D. Schmeidler, (1969), "The nucleolus of a characteristic function game", SIAM Journal of Applied Mathematics, 17(6), pp. 1163-1170.

[17] S.C. Littlechild, (1974), "A simple expression for the nucleolus in a special case", International Journal of Game Theory, 3(1), pp. 21-29.

[18] Électricité de France: Conditions générales de vente (CGV): http://entreprises.edf.fr/accueil/nos-offres-et-services-94896.html, (Web site consulted April 24, 2009)

[19] Hydro-Québec Tariff (M and G): http://www.hydroquebec.com/affaires. (Web site consulted April 24, 2009)

[20] Energy Information Administration, (2007), "Electric Power Annual 2006", U.S. Department of Energy

(See http://www.eia.doe.gov/cneaf/electricity/epa/epa_sum.html, (Web site consulted April 24, 2009))

[21] European Commission: Environment and energy statistics, (2006), "Electricity prices in the EU25 in January 2006", European Commission (See http://ec.europa.eu/eurostat/, (Web site consulted April 24, 2009)) 